\title{
Propensity Matched Analysis of Outcomes and Hospital Charges for Anterior versus Posterior Cervical Fusion for Cervical Spondylotic Myelopathy
}

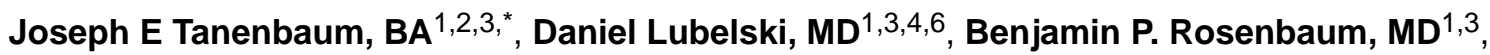 \\ Edward C. Benzel, MD ${ }^{1,3}$, and Thomas E Mroz, MD ${ }^{1,3,5}$ \\ ${ }^{1}$ Center for Spine Health, Cleveland Clinic, Cleveland, Ohio, USA \\ ${ }^{2}$ Case Western Reserve University School of Medicine, Cleveland, Ohio, USA \\ ${ }^{3}$ Department of Neurosurgery, Cleveland Clinic, Cleveland, Ohio, USA \\ ${ }^{4}$ Cleveland Clinic Lerner College of Medicine, Cleveland Clinic, Cleveland, Ohio, USA \\ ${ }^{5}$ Department of Orthopaedic Surgery, Cleveland Clinic, Cleveland, OH, USA \\ ${ }^{6}$ Department of Neurosurgery, Johns Hopkins University School of Medicine, Baltimore, MD
}

\begin{abstract}
STUDY DESIGN—Retrospective analysis of data from the Nationwide Inpatient Sample (NIS), a nationally representative, all-payer database of inpatient diagnoses and procedures in the United States.
\end{abstract}

OBJECTIVE-The objective of this study is to compare anterior cervical fusion (ACF) to posterior cervical fusion (PCF) in the treatment of cervical spondylotic myelopathy (CSM).

SUMMARY of BACKGROUND DATA-Previous studies used retrospective single-institution level data to quantify outcomes for CSM patients undergoing cervical fusion. It is unclear whether ACF or PCF is superior with regards to charges or outcomes for the treatment of CSM.

METHODS-We used NIS data to compare ACF to PCF in the management of CSM. All patients 18 years or older with a diagnosis of CSM between 1998-2011 were included. ACF patients were matched to PCF patients using propensity scores based on patient characteristics (number of levels fused, spine alignment, comorbidities), hospital characteristics, and patient demographics. Multivariable regression was used to measure the effect of treatment assignment on in-hospital charges, length of hospital stay, in-hospital mortality, discharge disposition, and dysphagia diagnosis.

RESULTS-From 1998-2011, we identified 109,728 hospitalizations with a CSM diagnosis. Of these patients, 45,629 (41.6\%) underwent ACF and 14,439 (13.2\%) underwent PCF. The PCF cohort incurred an average of $\$ 41,683$ more in-hospital charges $(\mathrm{p}<0.001$, inflation adjusted to 2011 dollars) and remained in hospital an average of 2.4 days longer $(\mathrm{p}<0.001)$ than the ACF

*Corresponding Author: Joseph Tanenbaum, Neurological Institute, Cleveland Clinic Center for Spine Health, Department of Neurosurgery, The Cleveland Clinic, 9500 Euclid Avenue, S-80, Cleveland, Ohio 44195, Tel: 518-369-1053,

joseph.tanenbaum@case.edu. 
cohort. The ACF cohort was just as likely to die in the hospital (OR 0.91, 95\% CI 0.68-1.2), 3.0 times more likely to be discharged to home or self-care (95\% CI 2.9-3.2), and 2.5 times more likely to experience dysphagia (95\% CI 2.0-3.1) than the PCF cohort.

CONCLUSIONS-In treating CSM, ACF led to lower hospital charges, shorter hospital stays, and an increased likelihood of being discharged to home relative to PCF.

\section{Keywords}

cervical discectomy; cervical fusion; dysphagia; propensity matching; Nationwide Inpatient Sample

\section{Introduction}

Two common surgical treatments for cervical spondylotic myelopathy (CSM) are anterior cervical fusion (ACF) and posterior cervical fusion (PCF). ${ }^{[1]} \mathrm{ACF}$ encompasses both discectomy and corpectomy. ACF and PCF procedures offer unique advantages and disadvantages. Although complications such as dysphagia and dysphonia are more common in ACF, the procedure enables direct decompression of cervical pathologies in kyphotic, neutral, or lordotic alignment. While PCF can be used in patients with neutral or lordotic spinal alignment, ${ }^{[2]}$ it can lead to significant postoperative muscle pain. ${ }^{[3-6]}$

Previous studies found that complication rates are higher with PCF than with ACF. ${ }^{[6]}$ In addition to traditional outcome measures such as complication rates, readmission rates, and revision surgery rates, recent studies comparing the two procedures focused on their costs. ${ }^{[7,8]}$ For example, Oglesby et al. found that from 2002-2009, the in-hospital cost of posterior cervical fusion increased more than twice as much as did the cost of anterior cervical fusion. ${ }^{[9]}$

Several previous studies used retrospective single-institution level data to quantify outcomes for CSM patients following anterior or posterior surgeries. ${ }^{[10-13]}$ It remains unclear whether ACF or PCF is superior with regards to cost effectiveness or outcomes for the treatment of CSM. One potential cause of this uncertainty is that a dearth of large-scale, randomized studies exists in the literature comparing outcomes associated with ACF and PCF when used to treat CSM.

The present study uses a large, multi-institutional, retrospectively collected, all-payer database of inpatient diagnoses and procedures. Using ACF and PCF cohorts, we sought to test our hypothesis, developed from previous non-randomized studies, ${ }^{[9,14]}$ that ACF leads to better outcomes at lower costs than PCF in the treatment of CSM.

\section{Methods}

\section{Data Collection}

This study used Nationwide Inpatient Sample (NIS) data from 1998-2011. Data points were chosen from hospitalizations if any diagnosis listed the International Classification of Disease, Ninth Revision, Clinical Modification (ICD-9-CM) diagnosis code for CSM 
(721.1). Patients were then grouped by treatment; grouping occurred between ACF and PCF, based on whether ICD-9-CM procedure codes 81.02 and 81.03, respectively, were listed for any procedure throughout an episode of in-hospital care. ${ }^{[15-17]}$

Established by the Agency for Healthcare Research and Quality (AHRQ), the NIS is the largest all-payer healthcare database in the United States. ${ }^{[18]}$ The NIS contains a $20 \%$ stratified sample of all hospital discharges from 1988-2011. Within the database, each entry corresponds to a single hospital admission. Using the NIS, national estimates can be generated by assigning weighted values to each hospital discharge. The NIS includes data on patient demographics, comorbidities, diagnoses, procedures performed, outcomes (e.g., length of hospital stay, hospital charges, mortality), and hospital features. ${ }^{[18]}$ Finally, the NIS codes admission diagnoses, procedures, and in-hospital complications using ICD-9-CM codes.

In order to mitigate bias, data were used starting in 1998 because the sampling strategy of the NIS changed that year. ${ }^{[17]}$ Furthermore, Elixhauser comorbidity data ${ }^{[19,20]}$ were collected beginning in 1998. The Elixhauser index is a composition of thirty comorbidities representing significant conditions associated with in-hospital mortality, including acute and chronic comorbidities. The Elixhauser index allows for standardized risk adjustment in administrative databases, and the NIS includes 29 of the AHRQ comorbidities originally discussed by Elixhauser et al. ${ }^{[19]}$

In addition, we obtained data on patient age, gender, race (White, Black, Hispanic, Native American, Asian, other), median income based on patient's zip code, primary payment type (Medicare, Medicaid, private insurance, self payment, no charge), hospital characteristics (academic hospital setting, admission source (emergency, urgent, or elective), weekend admission, hospital bed size, hospital region). We also obtained data on levels of fusion (either 2-3 levels fused or 4-8 levels fused) and spine alignment (kyphosis, lordosis, or neutral). Number of levels fused was determined using ICD-9-CM codes 81.62 and 81.63 (for 2-3 levels and 4-8 levels fused, respectively). ${ }^{[16,21]}$ Kyphosis was determined using ICD-9-CM codes 737.10 and 737.19 and lordosis was determined using ICD-9-CM codes 737.20 and 737.29.[16] Only patients aged eighteen years and older were included. IRB approval (Study \#14-1108) was obtained prior to start of study.

\section{Statistical Analysis}

Significant differences between surgical cohorts at baseline were determined using chi squared test for categorical data and Student $t$ test for discrete variables. An alpha level of less than 0.001 was used to denote statistical significance because of the large sample size included in the NIS database.

We generated matched cohorts using a propensity scoring method to minimize the effect of baseline characteristic imbalances between the ACF and PCF cohorts. We assigned a propensity score to each hospitalization based on the likelihood of treatment using a multivariable logistic regression that included number of levels fused, spine alignment, patient demographics, hospital characteristics, and payment source as covariates. Similar propensity scores between the two cohorts were matched using a nearest-neighbor method 
within .02 standard deviations of the calculated score without replacement. ${ }^{\text {[22-24] }}$

Furthermore, propensity scores were used to stratify the sample into five equally sized quintiles. Dividing a study population into quintiles using propensity scores can eliminate $90 \%$ of bias based on measured covariates. ${ }^{[25]}$

Following the work of Rosenbaum and Rubin, we used a standardized difference with an absolute value greater than 0.10 to determine significance in assessing balance across all measured covariates. ${ }^{[26]}$ The variables found to be unbalanced across the two cohorts following matching were included in the final regressions.

Outcome measures used to compare the ACF and PCF cohorts in both propensity matched and unmatched samples were LOS, hospital charges (adjusted for inflation to 2011 dollars), in-hospital mortality, dysphagia diagnosis (ICD-9-CM diagnosis code 787.2), and discharge disposition (discharge to home or to another care facility). Hospital charges were adjusted for inflation to 2011 dollars using the Bureau of Labor Statistics CPI Inflation Calculator. ${ }^{[27]}$ Discharge weights were used to generate national estimates. A multivariable linear regression model was used to measure the effect of treatment assignment and propensity score on LOS and hospital charges, whereas a multivariable logistic regression model was used to measure the effect of treatment assignment and propensity score on in-hospital mortality, discharge disposition, and dysphagia diagnosis. Analyses were performed using Stata software version 13.0 (Stata Corporation, College Station, TX, USA).

\section{Results}

From 1998-2011, the NIS includes 109,728 hospitalizations with a CSM diagnosis listed during an inpatient episode of care. Of these patients, 45,629 (41.6\%) underwent ACF and 14,439 underwent PCF (13.2\%). Patient demographics and comorbidities are summarized in Table 1.

Prior to matching, patients undergoing ACF were younger, more likely to be female, to be white, to have an elective admission, treated in a southern hospital, have private insurance, and to have been treated at a small or medium sized hospital relative to the PCF cohort. The PCF cohort was more likely to have been admitted on the weekend, to have an emergent or urgent admission, treated in a northeastern, midwestern, or teaching hospital, to pay with Medicare or Medicaid, and to be black or Hispanic. Finally, the PCF cohort was more likely to suffer from arthritis, congestive heart failure, chronic lung disease, depression, diabetes, hypertension, or liver disease, as determined by ICD-9 codes.

All covariates from Table 1 were used to generate a propensity score. We matched 11,671 PCF patients with ACF patients. We assessed the matched cohorts for covariate balance. As shown in Table 2, matching eliminated almost all significant differences in clinical characteristics, demographics, hospital characteristics, payment source, and comorbidity prevalence between the two cohorts with two exceptions: patients in the ACF cohort in the matched sample were more likely to be hypertensive and have diabetes mellitus than patients in the PCF cohort. Specifically, patients in the ACF and PCF cohorts were equally likely to have undergone multi-level fusion and were equally likely to be kyphotic or lordotic.

Clin Spine Surg. Author manuscript; available in PMC 2018 November 01. 
In the matched analysis, patients that underwent PCF incurred $\$ 41,683$ more in-hospital charges ( $\$ 59,934$ for ACF and $\$ 99,841$ for PCF, inflation adjusted to 2011 dollars, $\mathrm{p}<0.001$ ). Patients in the PCF cohort remained in hospital an average of 2.4 days longer than patients in the ACF cohort (4.3 days for ACF and 6.5 days for PCF, $\mathrm{p}<0.001$ ). The odds of inhospital mortality, however, were not statistically significantly different between the two cohorts $(0.80 \%$ for ACF and $.84 \%$ for PCF, OR $0.91,95 \%$ CI $0.68-1.21)$. As expected, the odds of experiencing dysphagia while in the hospital were 2.5 times greater in the ACF cohort relative to the PCF cohort (95\% CI 2.01-3.1). Lastly, the odds of being discharged to home were 3.0 times greater for the ACF cohort relative to the PCF cohort (95\% CI 2.93.2). These results are shown in Table 3.

We also performed a sensitivity analysis by first increasing and then decreasing the number of standard deviations used in the matching algorithm. These two analyses yielded similar results to those described above.

\section{Discussion}

Previous studies comparing outcomes between ACF and PCF have focused on reoperation rates, ${ }^{[28,29]}$ cost utility analyses, ${ }^{[8,30]}$ and in-hospital complication rates. ${ }^{[17]}$ For example, Oglesby et al. found that from 2002-2009, the in-hospital cost for PCF was more than twice as much as that of ACF. ${ }^{[9]}$ However, a recent review by Alvin et al. found that many cost studies in the ACF-PCF comparison literature fail to adequately include indirect costs in their analyses. ${ }^{[2]}$ Despite this limitation, Alvin et al. conclude that both dorsal and ventral decompression and fusion, when used to treat CSM, can lead to significant improvements in quality of life (QOL). Still, uncertainty remains about which of the two approaches is preferred in the treatment of CSM. The lack of randomized controlled trials comparing these two procedures has contributed to this uncertainty. Although the present study is not a randomized trial, the present study uses propensity matching of baseline factors among patients undergoing ACF and PCF to mitigate bias in evaluating in-hospital charges, length of hospital stay, in-hospital mortality, and discharge disposition. Following matching, the ACF and PCF cohorts were not significantly different with respect to baseline clinical characteristics, demographics, hospital characteristics, and payment source. The ACF cohort remained more likely to have diabetes mellitus and more likely to be hypertensive.

\section{In-Hospital Charges}

We found that the ACF cohort had an average of $\$ 41,683$ less in-hospital charges $(\$ 59,934$ for ACF and $\$ 99,841$ for PCF, inflation adjusted to 2011 dollars). While not a direct indicator of patient costs, hospital charges offer some insight into the financial burden patients and insurers confront. One partial contributor to the charge discrepancy is the significantly shorter LOS associated with ACF. A second source of the charge discrepancy is that PCF is often a longer procedure than ACF, leading to increased use of hospital resources.

Boakye et al. presented national estimates of complications and outcomes following spinal fusion for treatment of CSM. ${ }^{[14]}$ Using NIS data from 1993-2002, the authors identified patients with CSM and recorded the Elixhauser index, three co-morbidity variables, patient 
age, hospital size, and hospital volume. The authors performed multivariate logistic regression, controlling for the covariates described above. Boakye et al. found that ACF patients had significantly lower mean hospital charges than PCF patients $(\$ 23,309$ and $\$ 30,927$, respectively).

Although these estimates are lower than our estimates, Boakye et al. did not adjust their results for inflation. Moreover, the data in Boakye et al. extends only to 2002, when overall hospital charges were less for any hospitalization than during the time period included in our data set. Additionally, the ACF and PCF cohorts in the NIS are unbalanced across several variables at baseline, including clinically relevant details such as number of levels fused and spine alignment. Boakye et al. failed to include several of these variables in their study. The present study differs from Boakye et al. by using propensity matching to mimic the conditions of a randomized controlled trial. This matching technique allows for a more direct comparison of outcomes following ACF and PCF, specifically because we compared two cohorts with similar clinical characteristics. While hospital charges should not be relied upon to determine appropriate treatment approach and strategy, it is important to identify variations in charges among patients with similar covariate and demographic profiles that undergo surgery for similar pathologies.

\section{Length of Hospital Stay and Discharge Disposition}

The PCF cohort remained in hospital an average of 2.4 days longer than the ACF cohort. This is consistent with Oglesby et al. ${ }^{[9]}$ who used NIS data from 2002-2009 to assess trends in anterior and posterior cervical spine surgery for degenerative diseases and found that PCF patients had an average LOS significantly greater than that of anterior cervical fusion (ACF) patients (7.4 days for PCF compared to 2.5 days for ACF, $\mathrm{p}<.001$ ). They also found that PCF patients had greater mortality, comorbidities, costs, and longer hospitalizations compared to ACF patients. Similar to the limitations in Boakye et al., the Oglesby et al. study only described epidemiological trends in patients undergoing PCF and ACF. Without utilizing matching to produce cohorts that were balanced across covariates at baseline, Oglesby et al. could not directly compare ACF and PCF cohorts. In contrast, the matching technique used in the present study allows for a comparison of ACF and PCF cohorts that were similar across a range of covariates, including clinical characteristics and patient demographics.

In addition to shorter length of hospital stay, the ACF cohort had greater odds of being discharged to home or self-care compared to the PCF cohort (OR 3.0, 95\% CI 2.9-3.2). These findings suggest that ACF may require less intensive immediate post-discharge care than PCF.

Our results are consistent with Memtsoudis et al., ${ }^{[17]}$ which found that patients undergoing ACF were significantly more likely to be discharged home than patients undergoing PCF (92\% versus $61 \%$, respectively, $\mathrm{p}<.001$ ). Memtsoudis et al. analyzed NIS data for all instances of ACF and PCF use, not specifically for the treatment of CSM. Moreover, the authors call for a comparative analysis of ACF and PCF that uses age-matched cohorts similar to those in the present study. Finally, Boakye et al. found that $84 \%$ of patients undergoing spinal fusion (using either anterior or posterior surgical techniques) were 
discharged home. Although they provide descriptive statistics for adverse outcomes, the authors do not compare ACF and PCF patients on the basis of discharge disposition.

We also analyzed dysphagia incidence as a positive control to validate our data set. The reported incidence of dysphagia in the literature ranges from $1 \%-79 \%$ following anterior cervical spine surgery versus no such complication in posterior spine surgery. ${ }^{[31]}$ This is similar to our results, which showed that $2.5 \%$ of the ACF cohort and $1.0 \%$ of the PCF cohort developed dysphagia.

\section{Limitations and Strengths}

There are several limitations that should be considered when interpreting the results of this study. First, the NIS uses ICD-9-CM codes to report procedures and diagnoses. Codes are assigned based on a review of the clinician's notes. If a clinician omits a relevant detail in their documentation, relevant ICD-9-CM codes may be missed. Therefore, while useful, relying on these codes to determine our study population renders our outcomes subject to misclassification. ${ }^{[32]}$ Moreover, the ICD-9-CM codes we used for ACF and PCF (81.02 and 81.03 , respectively) do not include laminectomy or any mention of decompression, the latter of which is implied considering their use to treat CSM. No specific ICD-9-CM code exists for cervical laminectomy. Second, propensity matching never fully simulates the conditions of a randomized controlled trial, as only observed variables can be included in the matching algorithm. Unobserved (or unrecorded) variables may remain unbalanced and unaccounted for, even after matching. Prior to matching, the ACF and PCF cohorts were significantly different across several baseline demographics and characteristics. This analysis did not investigate the reason behind these differences, however we believe that this is an area worthy of future study. Following matching, however, our cohorts remained unbalanced across only two covariates: prevalence of diabetes mellitus and hypertension. The prevalence of each condition was higher in the ACF cohort than in the PCF cohort. Despite this difference, the ACF cohort had shorter LOS, lower hospital charges, and was more likely to be discharged to home. Third, the NIS does not include the exact number of vertebral levels involved in ACF or PCF procedures. Instead, the NIS records whether a patient underwent multi-level fusion (4-8 levels) or single-level fusion (2-3 levels). Fourth, neurologic status, functional outcomes, and other long-term clinical outcomes that would be useful for the purposes of comparing ACF and PCF are not present in administrative databases such as the NIS. Lastly, the NIS gathers administrative data from the inpatient episode of care and, therefore, conditions that may only become apparent with extended follow-up remain outside the scope of investigation.

Despite these limitations, our study mitigates bias similarly to a randomized trial by including a wide array of covariates in both our propensity matching algorithm and our final models. We were able to provide the results of a large cohort composed of patients from numerous institutions around the country, thereby generating more broadly generalizable results than any study on this topic to date. The results of this study further demonstrate the need for a prospective, randomized controlled trial comparing ACF to PCF in the treatment of CSM. 


\section{Conclusion}

Using a large, nationally representative sample of patient data comparing ACF to PCF, we found that ACF led to lower hospital charges, shorter hospital stays, and an increased likelihood of being discharged to home rather than to any other care setting. As the population ages, the incidence and prevalence of cervical spine degeneration among the elderly stands poised to increase with a concomitant increase in the incidence of CSM. In the current climate of dynamic changes in healthcare finance, procedures that lead to improved outcomes at lower charges become increasingly worthy of emphasis and recognition. Although our findings do not obviate the need for a randomized controlled trial with long-term follow-up, they represent another step toward determining the optimal surgical technique in the treatment of CSM.

\section{Acknowledgments}

The primary author was supported in part by NIH grant TL1 TR000441. No grants, technical, or corporate support were received by any other authors in conducting this study or writing this manuscript. No relevant conflicts of interest.

\section{References}

1. Wang MC, Kreuter W, Wolfla CE, Maiman DJ, Deyo RA. Trends and variations in cervical spine surgery in the United States: Medicare beneficiaries, 1992 to 2005. Spine (Phila Pa 1976). 2009; 34(9):955-61. discussion 962-3. [PubMed: 19352223]

2. Alvin MD, Lubelski D, Benzel EC, Mroz TE. Ventral fusion versus dorsal fusion: determining the optimal treatment for cervical spondylotic myelopathy. Neurosurg Focus. 2013; 35(1):E5.

3. Ghogawala Z, Martin B, Benzel EC, Dziura J, Magge SN, Abbed KM, Bisson EF, Shahid J, Coumans JV, Choudhri TF, Steinmetz MP, Krishnaney AA, King JT Jr, Butler WE, Barker FG 2nd, Heary RF. Comparative effectiveness of ventral vs dorsal surgery for cervical spondylotic myelopathy. Neurosurgery. 2011; 68(3):622-30. discussion 630-1. [PubMed: 21164373]

4. Yalamanchili PK, Vives MJ, Chaudhary SB. Cervical spondylotic myelopathy: factors in choosing the surgical approach. Adv Orthop. 2012; 2012:783762. [PubMed: 22312563]

5. Lad SP, Patil CG, Berta S, Santarelli JG, Ho C, Boakye M. National trends in spinal fusion for cervical spondylotic myelopathy. Surg Neurol. 2009; 71(1):66-9. discussion 69. [PubMed: 18514286]

6. Wang MC, Chan L, Maiman DJ, Kreuter W, Deyo RA. Complications and mortality associated with cervical spine surgery for degenerative disease in the United States. Spine (Phila Pa 1976). 2007; 32(3):342-7. [PubMed: 17268266]

7. King JT Jr, Abbed KM, Gould GC, Benzel EC, Ghogawala Z. Cervical spine reoperation rates and hospital resource utilization after initial surgery for degenerative cervical spine disease in 12,338 patients in Washington State. Neurosurgery. 2009; 65(6):1011-22. discussion 1022-3. [PubMed: 19934960]

8. Whitmore RG, Schwartz JS, Simmons S, Stein SC, Ghogawala Z. Performing a cost analysis in spine outcomes research: comparing ventral and dorsal approaches for cervical spondylotic myelopathy. Neurosurgery. 2012; 70(4):860-7. discussion 867. [PubMed: 21937935]

9. Oglesby M, Fineberg SJ, Patel AA, Pelton MA, Singh K. Epidemiological trends in cervical spine surgery for degenerative diseases between 2002 and 2009. Spine (Phila Pa 1976). 2013; 38(14): 1226-32. [PubMed: 23403550]

10. Edwards CC 2nd, Heller JG, Murakami H. Corpectomy versus laminoplasty for multilevel cervical myelopathy: an independent matched-cohort analysis. Spine (Phila Pa 1976). 2002; 27(11):116875. [PubMed: 12045513] 
11. Heller JG, Edwards CC 2nd, Murakami H, Rodts GE. Laminoplasty versus laminectomy and fusion for multilevel cervical myelopathy: an independent matched cohort analysis. Spine (Phila Pa 1976). 2001; 26(12):1330-6. [PubMed: 11426147]

12. Houten JK, Cooper PR. Laminectomy and posterior cervical plating for multilevel cervical spondylotic myelopathy and ossification of the posterior longitudinal ligament: effects on cervical alignment, spinal cord compression, and neurological outcome. Neurosurgery. 2003; 52(5):10817. discussion 1087-8. [PubMed: 12699550]

13. Ikenaga M, Shikata J, Tanaka C. Anterior corpectomy and fusion with fibular strut grafts for multilevel cervical myelopathy. J Neurosurg Spine. 2005; 3(2):79-85. [PubMed: 16370295]

14. Boakye M, Patil CG, Santarelli J, Ho C, Tian W, Lad SP. Cervical spondylotic myelopathy: complications and outcomes after spinal fusion. Neurosurgery. 2008; 62(2):455-61. discussion 461-2. [PubMed: 18382324]

15. Marawar S, Girardi FP, Sama AA, Ma Y, Gaber-Baylis LK, Besculides MC, Memtsoudis SG. National trends in anterior cervical fusion procedures. Spine (Phila Pa 1976). 2010; 35(15):14549. [PubMed: 20216341]

16. Nandyala SV, Marquez-Lara A, Fineberg SJ, Singh K. Comparison of revision surgeries for one- to two-level cervical TDR and ACDF from 2002 to 2011. Spine J. 2014; 14(12):2841-6. [PubMed: 24704499]

17. Memtsoudis SG, H A, Ma Y, Chiu YL, Sama AA, Girardi FP. Increased In-hospital Complications After Primary Posterior versus Primary Anterior Cervical Fusion. Clin Orthop. 2011; 469:649657. [PubMed: 20838946]

18. HCUP National Inpatient Sample (NIS). Healthcare Cost and Utilization Project (HCUP). Agency for Healthcare Research and Quality, R; MD: 1998-2011. http://www.hcup-us.ahrq.gov/ nisoverview.jsp. Accessed 12/15/14

19. Elixhauser A, S C, Harris DR, Coffey RM. Comorbidity Measures for Use with Administrative Data. Med Care. 1998; 36:8-27. [PubMed: 9431328]

20. Li B, E D, Faris P, Dean S, Quan H. Risk adjustment performance of Charlson and Elixhauser comorbidities in ICD-9 and ICD-10 administrative databases. BMC health services research. 2008; 8:12-12. 2007. [PubMed: 18194561]

21. Shamji MF, Cook C, Pietrobon R, Tackett S, Brown C, Isaacs RE. Impact of surgical approach on complications and resource utilization of cervical spine fusion: a nationwide perspective to the surgical treatment of diffuse cervical spondylosis. Spine J. 2009; 9(1):31-8. [PubMed: 18790678]

22. Austin PC. Balance diagnostics for comparing the distribution of baseline covariates between treatment groups in propensity-score matched samples. Stat Med. 2009; 28(25):3083-107. [PubMed: 19757444]

23. Austin PC, Mamdani MM. A comparison of propensity score methods: a case-study estimating the effectiveness of post-AMI statin use. Stat Med. 2006; 25(12):2084-106. [PubMed: 16220490]

24. Austin PC, Grootendorst P, Anderson GM. A comparison of the ability of different propensity score models to balance measured variables between treated and untreated subjects: a Monte Carlo study. Stat Med. 2007; 26(4):734-53. [PubMed: 16708349]

25. Rosenbaum PR, R D. Reducing Bias in Observational Studies Using Subclassification on the Propensity Score. Journal of the American Statistical Association. 1984

26. Rosenbaum PR, R D. Constructing a Control Group Using Multivariate Matched Sampling Methods That Incorporate the Propensity Score. The American Statistician. 1985

27. Consumer Price Index calculator. US Bureau of Labor Statistics Web site. http://www.bls.gov/cpi/. Accessed October 1

28. Veeravagu A, Cole T, Jiang B, Ratliff JK. Revision rates and complication incidence in single- and multilevel anterior cervical discectomy and fusion procedures: an administrative database study. Spine J. 2014; 14(7):1125-31. [PubMed: 24126076]

29. Singh K, P F, Park DK, Pelton MA, An HS, Goldberg EJ. Factors affecting reoperations after anterior cervical discectomy and fusion within and outside of a Federal Drug Administration investigational device exemption cervical disc replacement trial. The Spine Journal. 2012 
30. Fehlings MG, J N, Hewson SM, Massicotte EM, Kopjar B, Kalsi-Ryan S. Is surgery for cervical spondylotic myelopathy cost-effective? A cost-utility analysis based on data from the AOSpine North America prospective CSM study. Journal of Neurosurgery: Spine. 2012

31. Kalb S, Reis MT, Cowperthwaite MC, Fox DJ, Lefevre R, Theodore N, Papadopoulos SM, Sonntag VK. Dysphagia after anterior cervical spine surgery: incidence and risk factors. World Neurosurg. 2012; 77(1):183-7. [PubMed: 22155226]

32. Evans JM, M T. Misclassification and selection bias in case-control studies using an automated database. Pharmacoepidemiol Drug Safety. 1997 


\section{Table 1}

Baseline Characteristics and Demographics

\begin{tabular}{|c|c|c|c|}
\hline & $\operatorname{ACF}(N=45,629)$ & PCF $(N=14,439)$ & P-value \\
\hline Age (year) $\pm S D$ & $57.6 \pm 12.1$ & $62.6 \pm 12.0$ & $<0.001^{*}$ \\
\hline \multicolumn{4}{|l|}{ Admission Type } \\
\hline Elective & $77.00 \%$ & $71.80 \%$ & $<0.001^{*}$ \\
\hline Emergent & $5.75 \%$ & $9.52 \%$ & $<0.001^{*}$ \\
\hline Urgent Care & $6.87 \%$ & $7.89 \%$ & $<0.001$ * \\
\hline Weekend Admission & $2.58 \%$ & $4.06 \%$ & $<0.001^{*}$ \\
\hline Elixhauser Index Score \pm SD & $1.4 \pm 1.4$ & $2.0 \pm 1.6$ & $<0.001^{*}$ \\
\hline Female & $46.70 \%$ & $39.90 \%$ & $<0.001^{*}$ \\
\hline \multicolumn{4}{|l|}{ Hospital Sizeize } \\
\hline Small & $10.90 \%$ & $8.40 \%$ & $<0.001$ * \\
\hline Medium & $21.90 \%$ & $18.00 \%$ & $<0.001$ * \\
\hline Large & $66.80 \%$ & $73.00 \%$ & $<0.001^{*}$ \\
\hline \multicolumn{4}{|l|}{ Hospital Region } \\
\hline Northeast & $12.70 \%$ & $19.70 \%$ & $<0.001^{*}$ \\
\hline Midwest & $19.80 \%$ & $21.40 \%$ & $<0.001^{*}$ \\
\hline South & $47.30 \%$ & $39.70 \%$ & $<0.001^{*}$ \\
\hline East & $20.20 \%$ & $19.20 \%$ & $<0.05$ \\
\hline Teaching Hospital & $55.60 \%$ & $71.20 \%$ & $<0.001^{*}$ \\
\hline \multicolumn{4}{|l|}{ Primary Payment Method } \\
\hline Medicare & $34.50 \%$ & $50.00 \%$ & $<0.001^{*}$ \\
\hline Medicaid & $5.90 \%$ & $6.80 \%$ & $<0.001^{*}$ \\
\hline Private Insurance & $50.70 \%$ & $35.90 \%$ & $<0.001^{*}$ \\
\hline Self-Payment & $1.70 \%$ & $1.90 \%$ & 0.08 \\
\hline No-Charge & $0.18 \%$ & $0.30 \%$ & 0.07 \\
\hline Other & $7.00 \%$ & $5.00 \%$ & $<0.001^{*}$ \\
\hline \multicolumn{4}{|l|}{ Race } \\
\hline Asian & $1.20 \%$ & $1.50 \%$ & $<0.01$ \\
\hline Black & $9.80 \%$ & $12.50 \%$ & $<0.001^{*}$ \\
\hline Hispanic & $3.80 \%$ & $4.90 \%$ & $<0.001^{*}$ \\
\hline White & $62.10 \%$ & $59.90 \%$ & $<0.001^{*}$ \\
\hline Native American & $0.37 \%$ & $0.40 \%$ & 0.621 \\
\hline Other & $1.60 \%$ & $2.00 \%$ & $<0.001^{*}$ \\
\hline \multicolumn{4}{|l|}{ Comorbidity } \\
\hline AIDS & $0.7 \%$ & $0.13 \%$ & $<0.05$ \\
\hline Alcohol Use & $1.80 \%$ & $3.10 \%$ & $<0.001^{*}$ \\
\hline
\end{tabular}

Clin Spine Surg. Author manuscript; available in PMC 2018 November 01. 


\begin{tabular}{cccc}
\hline & ACF (N=45,629) & PCF (N=14,439) & P-value \\
Arthritis & $2.70 \%$ & $5.60 \%$ & $<0.001^{*}$ \\
Congestive Heart Failure & $2.10 \%$ & $4.00 \%$ & $<0.001^{*}$ \\
Chronic Lung Disease & $15.50 \%$ & $18.30 \%$ & $<0.001^{*}$ \\
Depression & $9.80 \%$ & $11.10 \%$ & $<0.001^{*}$ \\
Diabetes Mellitus & $15.10 \%$ & $17.90 \%$ & $<0.001^{*}$ \\
Hypertension & $46.10 \%$ & $57.50 \%$ & $<0.001^{*}$ \\
Obese & $7.70 \%$ & $8.00 \%$ & 0.32 \\
Liver Disease & $1.00 \%$ & $1.80 \%$ & $<0.001^{*}$ \\
Multi-Level Fusion (4-8 levels) & $13.60 \%$ & $12.80 \%$ & $<0.10$ \\
Kyphosis & $2.10 \%$ & $5.90 \%$ & $<0.001^{*}$ \\
Lordosis & $0.20 \%$ & $0.50 \%$ & $<0.001^{*}$ \\
Median Income in Zip Code (quartile) & & & $<0.001^{*}$ \\
$1^{\text {st }}$ & $19.40 \%$ & $21.90 \%$ & $<0.05$ \\
$2^{\text {nd }}$ & $24.70 \%$ & $23.80 \%$ & 0.139 \\
$3^{\text {rd }}$ & $25.90 \%$ & $25.30 \%$ & $<0.05$ \\
$4^{\text {th }}$ & $27.40 \%$ & $26.30 \%$ & \\
\hline
\end{tabular}

* denotes statistically significantly different result

All data come from the NIS. All patients with a diagnosis of CSM between 1998-2011 and that were 18 years or older at the time of admission were included. The Elixhauser Index is a composition of thirty comorbidities characterizing significant conditions associated with in-hospital mortality, including acute and chronic comorbidities. The Elixhauser index allows for standardized risk adjustment in administrative databases such as the NIS. The Elixhauser Index Score ranges from zero to thirty, with zero indicating that a patient has none of the thirty comorbidities and thirty indicating that a patient has all thirty of the comorbidities. An alpha-level of 0.001 was used to determine significance. All calculations were performed using Stata 13.0. 
Table 2

Baseline Characteristics and Demographics for Matched Cohorts

\begin{tabular}{|c|c|c|c|}
\hline & $\operatorname{ACF}(N=11,671)$ & $\operatorname{PCF}(\mathrm{N}=11,671)$ & Standardized Difference \\
\hline \multicolumn{4}{|l|}{ Age } \\
\hline Under 30 & $1 \%$ & $1 \%$ & 0.001 \\
\hline Under 40 & $3 \%$ & $3 \%$ & -0.01 \\
\hline Under 50 & $14 \%$ & $16 \%$ & -0.065 \\
\hline Under 60 & $38 \%$ & $42 \%$ & -0.092 \\
\hline Under 70 & $67 \%$ & $71 \%$ & -0.079 \\
\hline Under 80 & $91 \%$ & $92 \%$ & -0.027 \\
\hline \multicolumn{4}{|l|}{ Admission Type } \\
\hline Elective & $69 \%$ & $70 \%$ & -0.02 \\
\hline Emergent & $12 \%$ & $11 \%$ & 0.022 \\
\hline Urgent Care & $9 \%$ & $9 \%$ & 0.003 \\
\hline Weekend Admission & $5 \%$ & $5 \%$ & -0.003 \\
\hline \multicolumn{4}{|l|}{ Elixhauser Index Score } \\
\hline 0 & $16 \%$ & $18 \%$ & -0.07 \\
\hline 1 & $25 \%$ & $26 \%$ & -0.022 \\
\hline 2 & $26 \%$ & $25 \%$ & 0.023 \\
\hline 3 or more & $33 \%$ & $31 \%$ & 0.056 \\
\hline Female & $39 \%$ & $41 \%$ & -0.048 \\
\hline \multicolumn{4}{|l|}{ Hospital Bedsize } \\
\hline Small & $10 \%$ & $10 \%$ & -0.002 \\
\hline Medium & $20 \%$ & $20 \%$ & -0.015 \\
\hline Large & $71 \%$ & $70 \%$ & 0.015 \\
\hline \multicolumn{4}{|l|}{ Hospital Region } \\
\hline Northeast & $21 \%$ & $20 \%$ & 0.043 \\
\hline Midwest & $22 \%$ & $21 \%$ & 0.012 \\
\hline South & $38 \%$ & $39 \%$ & -0.039 \\
\hline East & $19 \%$ & $20 \%$ & -0.009 \\
\hline Teaching Hospital & $73 \%$ & $71 \%$ & 0.055 \\
\hline \multicolumn{4}{|c|}{ Primary Payment Method } \\
\hline Medicare & $54 \%$ & $49 \%$ & 0.089 \\
\hline Medicaid & $8 \%$ & $8 \%$ & 0.017 \\
\hline Private & $30 \%$ & $35 \%$ & -0.103 \\
\hline Self & $2 \%$ & $2 \%$ & 0.012 \\
\hline No Charge & $0 \%$ & $0 \%$ & 0.008 \\
\hline Other & $6 \%$ & $6 \%$ & -0.014 \\
\hline \multicolumn{4}{|l|}{ Race } \\
\hline Asian & $2 \%$ & $2 \%$ & 0.019 \\
\hline Black & $14 \%$ & $14 \%$ & 0.005 \\
\hline Hispanic & $6 \%$ & $6 \%$ & 0.016 \\
\hline
\end{tabular}




\begin{tabular}{|c|c|c|c|}
\hline & $\operatorname{ACF}(N=11,671)$ & PCF $(N=11,671)$ & Standardized Difference \\
\hline Native American & $0 \%$ & $0 \%$ & 0.002 \\
\hline White & $2 \%$ & $2 \%$ & -0.001 \\
\hline Other & $56 \%$ & $56 \%$ & -0.016 \\
\hline \multicolumn{4}{|l|}{ Comorbidity } \\
\hline AIDS & $0 \%$ & $0 \%$ & -0.005 \\
\hline Alcohol Use & $3 \%$ & $3 \%$ & -0.013 \\
\hline Arthritis & $4 \%$ & $5 \%$ & -0.075 \\
\hline $\mathrm{CHF}$ & $4 \%$ & $4 \%$ & 0.021 \\
\hline Chronic Lung Disease & $20 \%$ & $18 \%$ & 0.059 \\
\hline Depression & $12 \%$ & $11 \%$ & 0.031 \\
\hline Diabetes & $23 \%$ & $18 \%$ & $0.129^{*}$ \\
\hline Hypertension & $62 \%$ & $56 \%$ & $0.111^{*}$ \\
\hline Liver Disease & $1 \%$ & $2 \%$ & -0.029 \\
\hline Obesity & $9 \%$ & $8 \%$ & 0.044 \\
\hline Multi-level Fusion (4-8 levels) & $15 \%$ & $15 \%$ & -0.007 \\
\hline Kyphosis & $6 \%$ & $6 \%$ & -0.011 \\
\hline Lordosis & $1 \%$ & $1 \%$ & -0.01 \\
\hline \multicolumn{4}{|c|}{ Median Income in Zip Code (quartile) } \\
\hline $1^{\text {st }}$ & $23 \%$ & $23 \%$ & -0.003 \\
\hline $2^{\text {nd }}$ & $24 \%$ & $24 \%$ & -0.015 \\
\hline $3^{\text {rd }}$ & $25 \%$ & $25 \%$ & -0.001 \\
\hline $4^{\text {th }}$ & $25 \%$ & $25 \%$ & 0.014 \\
\hline
\end{tabular}

denotes statistically significantly different result

A matched sample was generated using a propensity-matching algorithm to match patients undergoing ACF to those undergoing PCF for treatment of CSM. All patients were 18 or older. All data are from the NIS from 1998-2011. The Elixhauser Index is a composition of thirty comorbidities characterizing significant conditions associated with in-hospital mortality, including acute and chronic comorbidities. The Elixhauser index allows for standardized risk adjustment in administrative databases such as the NIS. The Elixhauser Index Score ranges from zero to thirty, with zero indicating that a patient has none of the thirty comorbidities and thirty indicating that a patient has all thirty of the comorbidities. Following the work of Rosenbaum and Rubin (1985), a standardized difference with an absolute value greater than 0.10 was used to determine significance. 


\section{Table 3}

\section{Outcomes Following ACF Relative To PCF}

\begin{tabular}{lcc}
\hline & ACF (95\% CI) & p-value \\
\cline { 2 - 3 } Hospital Charges (Inflation Adjusted to 2011 USD) & $-41,683(-43,596--39,770)$ & $<0.001^{*}$ \\
LOS (Days) & $-2.42(-2.60--2.24)$ & $<0.001^{*}$ \\
Mortality (OR) & $0.91(0.68-1.21)$ & 0.512 \\
Home Discharge (OR) & $3.0(2.9-3.2)$ & $<0.001^{*}$ \\
Dysphagia (OR) & $2.53(2.04-3.14)$ & $<0.001^{*}$ \\
\hline * & &
\end{tabular}

A matched sample was generated using a propensity-matching algorithm to match patients undergoing ACF to those undergoing PCF for treatment of CSM. All patients were 18 years or older. All data come from the NIS. Hospital charges are reported in 2011 USD and length of stay (LOS) is reported in days. Mortality, discharge disposition (either to home or to another care facility), and incidence of dysphagia are reported as odds ratios. An alpha-level of 0.001 was used to determine significance. All outcomes are reported with $95 \%$ CI. Outcomes are robust to heteroskedasticity. All calculations were performed using Stata 13.0. 